\title{
Effects of Modified Ohm's and Fourier's Laws on Electromagnetic Micropolar Fluid Subjected to Ramp-Type Heating
}

\author{
M. Zakaria ${ }^{1,2}$ \\ ${ }^{1}$ Mathematics department, Faculty of Education, El-Shatby Alexandria University, Alexandria, 21526, Egypt \\ ${ }^{2}$ Department of Mathematics, Faculty of Science, Al-Baha University, P.O. Box 1988, Al-Baha Kingdom of Saudi Arabia
}

\begin{abstract}
Effects of modified Ohm's and Fourier's laws on two-dimensional electromagnetic (EM) flow of an incompressible micropolar fluid over a moving plate are studied. Ohm's law is modified by the inclusion of two terms, one for the temperature gradient and the other for the cross product of the velocity with the initial magnetic field. Fourier's law of heat conduction is modified to include the relaxation time. The effects of the modified Ohm's law (ko parameter) and Alfven velocity parameter $\alpha$ in two cases the first for strong concentration $(n=0)$ and the other for weak concentration $(n=1 / 2)$ have discussed. The purpose of the latter term is to produce finite speed of heat conduction. Laplace and exponential Fourier transform techniques are used to obtain the solution by a direct approach. The solution of the problem in the physical domain is obtained by using a numerical method for the inversion of the Laplace transforms based on Fourier series expansions. The distributions of the velocity components, the temperature, and the induced magnetic and electric fields are obtained. The numerical values of these functions are represented graphically.
\end{abstract}

Keywords Micropolar, Electromagnetic, Modified Ohm's and Fourier's Laws, Seebeck Effect

\section{Introduction}

The physical mechanisms of heat, mass, and momentum transport in small-scale units may differ significantly from those in macroscale equipment[1,2]. Fundamental and applied investigations of microscale phenomena in fluid mechanics are motivated by developments in the areas of biological molecular machinery, atherogenesis, microcirculation, and microfluidics. At scales larger than a micron, the fluid can be treated as a continuum, and the flow is governed by the Navier-Stokes equation. The continuum model assumes that the properties of the material vary continuously throughout the flow domain. In Newtonian continuum mechanics, the fluid is modeled as a dense aggregate of particles, possessing mass, and translational velocity. However, the field equation, such as the Navier-Stokes equation, does not account for the rotational effects of the fluid micro- constituents.

In the theory of micropolar fluids[3], rigid particles contained in a small volume element can rotate about the centroid of the volume element. The rotation is described by an independent micro-rotation vector. Micropolar fluids can

\footnotetext{
* Corresponding author:

zakariandm@yahoo.com (M. Zakaria)

Published online at http://journal.sapub.org/mechanics

Copyright (C) 2012 Scientific \& Academic Publishing. All Rights Reserved
}

support body couples and exhibit microrotational effects. The theory of micropolar fluids has shown promise for predicting fluid behaviour at microscale. Papautsky et al.[1] found that a numerical model for water flow in microchannels based on the theory of micropolar fluids gave better predictions of experimental results than those obtained using the Navier-Stokes equation. Micropolar fluids can model anisotropic fluids, liquid crystals with rigid molecules, magnetic fluids, clouds with dust, muddy fluids, and some biological fluids[3]. In view of their potential application in microscale fluid mechanics and non-Newtonian fluid mechanics, it is worth exploring new fundamental solutions.

Srinivas and Kothandapani[4] studied the influence of heat and mass transfer on MHD peristaltic flow through a porous space with compliant walls. Chen[5] performed a magneto-hydrodynamic mixed convection of a power-law fluid past a stretching surface in the presence of thermal radiation and internal heat generation/absorption. Many authors have extended the above model to the flow of micropolar fluid past an infinite horizontal moving plate, in the presence of a magnetic under different physical situations (see for instance[6-10]). Norzieha et al.[11] have analyzed the MHD flow of Stokes' second problem for an MHD second grade fluid in a porous space. Mostafa[12] examined the same problem with Thermal radiation effect. Ezzat et al.[13] studied the combined heat and mass transfer for unsteady MHD flow of perfect conducting micropolar fluid with 
thermal relaxation. Turkyilmazoglu[14] have analyzed the effects of uniform radial electric field on the MHD heat and fluid flow due to a rotating disk Original Research Article. Notable work in this field were the work of Das[15] offered an approximation method for the Effect of chemical reaction and thermal radiation on heat and mass transfer flow of MHD micropolar fluid in a rotating frame of reference.

All the above-mentioned investigations are restricted to only MHD micropolar fluid flow analysis. No efforts have been made to study the effects of induced magnetic and electric fields on micropolar fluid flow over a moving plate. Yet, a wide variety of industrial applications requires serious consideration of electromagnetic (EM) effects in fluid. For example, electordynamic applications include pumping and levitation of liquids and gases, extractions of contaminants from gases such as smokes or liquids such as slurries. It has applications in the fields of polyelectrolytes (e.g., DNA), electrohydrodynamic spraying, and charge distribution in the atmosphere during thunderstorms. In the area of magnetic application, we have magnetohydrodynamics (MHD), magnetic fluids, and plasma. In contrast to the classical discipline (MHD), the theory of micropolar fluids predicts many other phenomena not covered by the classical theory. For example, motions of magnetic bubbly fluids and fluids with large numbers of small vortices or suspensions (e.g., dirty gases and fluids) cannot be treated by means of the classical theory of MHD[16].

The effects of induced magnetic and electric fields on micropolar fluid problems have extensively studied by Zakaria[17], Ezzat and Zakaria[18], Zakaria[19], Ezzat et. $\mathrm{Al}[20]$ carried out an analysis to study Magneto- hydrodynamic boundary layer flow past a stretching plate and heat transfer.

In a thermoelectric material there are free electrons or holes which carry both charge and heat. To a first approximation, the electrons and holes in a thermoelectric semiconductor behave like a gas of charged particles. If a normal (uncharged) gas is placed in a box within a temperature gradient, where one side is cold and the other is hot, the gas molecules at the hot end will move faster than those at the cold end. The faster hot molecules will diffuse further than the cold molecules and so there will be a net build up of molecules (higher density) at the cold end. The density gradient will drive the molecules to diffuse back to the hot end. In the steady state, the effect of the density gradient will exactly contracts the effect of the temperature gradient so there is no net flow of molecules. If the molecules are charged, the buildup of charge at the cold end will also produce a repulsive electrostatic force (and therefore electric potential) to push the charges back to the hot end. The electric potential (Voltage) produced by a temperature difference is known as the Seebeck effect and the proportionality constant is called the Seebeck coefficient.

The objective of this paper is to consider two dimensional electromagnetic micropolar fluid subjected to ramp-type heating taken into consideration the effects of modified Ohm's and Fourier's laws. Ohm's law is modified by the inclusion of two terms, one for the temperature gradient (Seebeck effect) and the other for the cross product of the velocity with the initial magnetic field. Fourier's law of heat conduction is modified to include the relaxation time. The formulas of the velocity components, the temperature, and the induced magnetic and electric fields are obtained. The ramp-type heating application is applied to our problem to get the solution in the complete form. The considered variables are presented graphically and comparisons and discussions are made.

\section{Basic Equations}

Here we consider a conducting unsteady flow of an incompressible MHD micropolar conducting fluid, with modified Ohm's law, of finite conductivity $\sigma_{0}$, permeated by an initial magnetic field $\mathbf{H}$. This produces an induced magnetic field $\mathrm{h}$ and induced electric field $\mathbf{E}$.

The basic equations of magnetic and electric fields, continuity, motion, and energy for unsteady flow of micropolar fluids in the presence of a transverse magnetic field with the velocity vector $\mathbf{V}$ and the rotation vector $\boldsymbol{\omega}$ are:

1. The variation of the magnetic and electric fields are given by Maxwell's equations

$$
\begin{array}{r}
\text { curl } h=J+\frac{\partial D}{\partial t}, \\
\operatorname{curl} E=-\frac{\partial B}{\partial t}, \\
\nabla \cdot B=0, \quad \nabla \cdot D=\rho_{e}, \\
B=\mu_{o} h, \quad D=\varepsilon_{o} E
\end{array}
$$

And the modified Ohm's law[21,22]:

$$
J=\sigma_{o}[E+V \times B]+\rho_{e} V-k_{o} \nabla T,
$$

Where $k_{o}=\sigma_{o} S$ the coefficient connecting the temperature gradient and electric current and $\mathrm{S}$ is the Seebeck coefficient

2. The continuity equation has the form:

$$
\frac{\partial \rho}{\partial \mathrm{t}}+\operatorname{div} \rho \mathrm{V}=0
$$

3. The equations of motion have the form

$$
\begin{gathered}
\rho \frac{D V}{D t}=\rho b-\nabla p-\left(\mu+\mu_{r}\right) \nabla \times \nabla \times V+\mu_{r} \nabla \times \omega \\
+\left(\lambda_{1}+2 \mu+\mu_{r}\right) \nabla(\nabla \cdot V)+\mu_{0}\left(\mathrm{~J} \times \mathrm{H}_{0}\right), \\
\rho j \frac{D \omega}{D t}=\mu_{r}(\nabla \times V-2 \omega)-\gamma \nabla \times \nabla \times \omega+\left(\alpha_{1}+\beta+\gamma\right) \nabla(\nabla \cdot \omega)+\rho \mathrm{I},(8)
\end{gathered}
$$

where the material constants $\lambda_{1}, \mu, \mu_{\mathrm{r}}$ are the viscosity coefficients and $\alpha_{1}, \beta, \gamma$ are the gyroviscosity coefficients. These constants confirm to the inequalities

$$
\begin{array}{lll}
\mu_{r} \geq 0, & 2 \mu+\mu_{r} \geq 0, & 3 \lambda_{1}+2 \mu+\mu_{3} \geq 0 . \\
\gamma \geq 0, & |\beta| \leq \gamma, & 3 \alpha_{1}+\beta+\gamma \geq 0 .
\end{array}
$$

4. The equation of energy in the absence of heat source is given by: 


$$
\rho T_{o} \frac{D \eta}{D t}=-\nabla \cdot q
$$

The entropy $\eta$ may be written in terms of temperature as follows

$$
\rho \eta=\frac{\rho C_{E}}{T_{o}}\left(T-T_{o}\right),
$$

The generalized Fourier's law is given by

$$
q+\tau_{o} \frac{\partial q}{\partial t}=-\lambda \nabla T
$$

where the convective derivative

$$
\frac{\mathrm{D}}{\mathrm{Dt}} \text { defined as } \frac{\mathrm{D}}{\mathrm{Dt}}=\frac{\partial}{\partial t}+(V \cdot \nabla) \text {. }
$$

By eliminating $\eta$ between (9) and (10) and using (11) we get the equation of heat conduction for the linear theory as follows

$$
\lambda \nabla^{2} T=\rho C_{E}\left(\frac{\partial}{\partial t}+\tau_{0} \frac{\partial}{\partial t}\right) \frac{D T}{D t},
$$

\section{Formulation of the Problem}

Our interest here is the case of unsteady two-dimensional flow of an incompressible micropolar conducting fluid past an infinite horizontal moving plate, with modified Ohm's and Fourier's laws permeated by an initial magnetic field $\mathrm{H}_{\mathrm{o}}$, acting along the $\mathrm{z}$-axis. The velocity components of the fluid are $\mathrm{u}$, and $\mathrm{v}$ in the $\mathrm{x}$ and $\mathrm{y}$ directions, respectively, taken parallel and perpendicular to the plate. We assume the initial magnetic field $\mathbf{H}$, the induced magnetic field, the induced electric field $\mathbf{E}$ which is normal to the considered magnetic field, and the electric current density $\mathbf{J}$ is parallel to the electric field as $\mathbf{H}=\left(0,0, H_{0}\right), \mathbf{h}=(0,0, h), \mathbf{E}=\left(E_{1}, E_{2}, 0\right)$, and $\mathbf{J}=\left(\mathrm{J}_{1}, \mathbf{J}_{2}, 0\right)$, and the microrotation in $\mathrm{z}$ - axis $\boldsymbol{\omega}=(0,0$, $\omega)$.

Since the system (1)-(8) and (11) is nonlinear; we come across certain difficulties during its investigation. On the other hand, to solve many problems of applied nature it is sufficient to consider a linearized variant of the system (1)-(8). The equations of Navier-Stokes can be linearized by two well-known methods: that of Stokes and that of Oseen. When using Stokes' method of linearization, nonlinear terms are totally discarded. This method yields satisfactory results for small $\mathbf{V}, \boldsymbol{\omega}$ and $\mathrm{T}$ (note that in this case nonlinear terms are small values of higher order). However, if the fluid flow velocity $\mathbf{V}$ is not a small value, this model leads to an essential error. In particular, the effects predicted by this method when a fluid flows along a solid body do not agree with experimental data.

A lesser error is obtained in the case of linearization by Oseen's method consisting in the following: it is assumed that fluid flow differs but little from flow along the $\mathrm{x}$-axis with constant velocity $\mathrm{v}_{0}$.

$$
\text { Then we set }(V \cdot \nabla) \approx v_{o} \frac{\partial}{\partial x}[23,24] \text {. }
$$

Under these assumptions, the vector system (1)-(8) and (11) reduces to an Oseen-linearized system of scalar equations of a MHD micropolar conducting fluid with modified Ohm's law in two-dimensional case:

$$
\begin{aligned}
& \frac{\partial u}{\partial x}+\frac{\partial v}{\partial y}=0, \\
& \frac{\partial u}{\partial t}+\frac{\partial u}{\partial x}=-\frac{\partial p}{\partial x}+(1+\chi) \nabla^{2} u+\chi \frac{\partial \omega}{\partial y}+\alpha^{2} \mathrm{v}_{1} \mathrm{~J}_{2}, \\
& \frac{\partial v}{\partial t}+\frac{\partial v}{\partial x}=-\frac{\partial p}{\partial y}+(1+\chi) \nabla^{2} v-\chi \frac{\partial \omega}{\partial x}-\alpha^{2} \mathrm{v}_{1} \mathrm{~J}_{1} \\
& \frac{\partial \omega}{\partial t}+\frac{\partial \omega}{\partial x}=\lambda_{1} \nabla^{2} \omega-2 \sigma \omega-\sigma \nabla^{2} \Phi, \\
& \left(1+\tau_{o} \frac{\partial}{\partial t}\right)\left(\frac{\partial T}{\partial t}+\frac{\partial T}{\partial x}\right)=\frac{1}{P_{r}} \nabla^{2} T \\
& \frac{\partial h}{\partial y}=v_{1} J_{1}+\frac{v_{o}^{2}}{c^{2}} \frac{\partial E_{1}}{\partial t} \\
& \frac{\partial h}{\partial x}=-v_{1} J_{2}-\frac{v_{o}^{2}}{c^{2}} \frac{\partial E_{2}}{\partial t} \\
& \frac{\partial E_{1}}{\partial y}-\frac{\partial E_{2}}{\partial x}=\frac{\partial h}{\partial t} \\
& J_{1}=\left[E_{1}+v\right]-\mathrm{k}_{\mathrm{o}} \frac{\partial T}{\partial x}, \\
& J_{2}=\left[E_{2}-u\right]-\mathrm{k}_{\mathrm{o}} \frac{\partial T}{\partial y},
\end{aligned}
$$

where $v_{1}=\mu \mu_{o} \sigma_{o}$.

For simplifications the above equations we have used the following non-dimensional variables (dropping the asterisks for convenience):

$$
\begin{aligned}
& x^{*}=\frac{v_{o}}{v} x, \quad y^{*}=\frac{v_{o}}{v} y, \quad t^{*}=\frac{v_{o}^{2}}{v} t, \quad \tau_{o}^{*}=\frac{v_{o}^{2}}{v} t_{o}, \quad T^{*}=\frac{T-T_{\infty}}{T_{o}}, \\
& u^{*}=\frac{u}{v_{o}}, \quad v^{*}=\frac{v}{v_{o}}, \quad \omega^{*}=\frac{v \omega}{v_{o}^{2}}, \quad E_{i}^{*}=\frac{E_{i}}{v_{o} \mu_{o} H_{o}}, \quad \chi=\frac{v_{r}}{v} \\
& J_{i}^{*}=\frac{J_{i}}{v_{o} \sigma_{o} \mu_{o} H_{o}}, \quad h^{*}=\frac{h}{H_{o}}, \quad \alpha^{*}=\frac{\alpha}{v_{o}}, \quad k_{o}^{*}=\frac{T_{o}}{H} k_{0}, \quad p^{*}=\frac{v_{o}}{v} p, \\
& \sigma=\frac{v v_{r}}{j v_{o}^{2}}, \quad \lambda_{1}=\frac{\gamma}{\rho j}, \quad P_{r}=\frac{\rho C_{p} v}{\lambda}, \quad k_{o}^{*}=\frac{T_{o} k_{o}}{\sigma_{o} \mu_{o} H_{o} v} .
\end{aligned}
$$

Differentiating Eq. (14) with respect to $\mathrm{x}$ and Eq. (15) with respect to $\mathrm{y}$, and then adding these two equations, we obtain

$$
p=-\alpha^{2} \mathrm{v}_{1} \xi
$$

Differentiating Eq. (14) with respect to y and Eq. (15) with respect to $\mathrm{x}$, and then subtracting these two equations, we obtain

$$
\frac{\partial \Phi}{\partial t}+\frac{\partial \Phi}{\partial x}=(1+\chi) \nabla^{2} \Phi+\chi \omega+\alpha^{2} \mathrm{v}_{1} \varsigma,
$$

Differentiating Eq. (21) with respect to $x$ and Eq. (22) with respect to $\mathrm{y}$, and then adding these two equations then using Eqs. (18)-(20), we obtain 


$$
\left(\frac{\partial}{\partial t}+\frac{v_{1} c^{2}}{v_{o}^{2}}\right) \varsigma=-\frac{\partial \Phi}{\partial t}-k_{o} \frac{\partial T}{\partial t},
$$

Differentiating Eq. (21) with respect to y and Eq. (22) with respect to $\mathrm{x}$, and then subtracting these two equations then using Eqs. (18)-(20), we obtain

$$
\left(\nabla^{2}-\frac{v_{o}^{2}}{c^{2}} \frac{\partial^{2}}{\partial^{2} t}-v_{1} \frac{\partial}{\partial t}\right) \xi=0, .
$$

where $\nabla^{2}=\frac{\partial^{2}}{\partial x^{2}}+\frac{\partial^{2}}{\partial y^{2}}$ is a two dimensional Laplace operator, $\Phi$ is the stream function satisfying the continuity equation with

$$
u=\frac{\partial \Phi}{\partial y}, \quad v=-\frac{\partial \Phi}{\partial x}, \quad \nabla^{2} \Phi=\frac{\partial u}{\partial y}-\frac{\partial v}{\partial x},
$$

and $\zeta, \xi$ the potential functions of current density defined by

$$
\begin{aligned}
& J_{1}=\frac{\partial \varsigma}{\partial x}+\frac{\partial \xi}{\partial y}, \quad J_{2}=\frac{\partial \varsigma}{\partial y}-\frac{\partial \xi}{\partial x} \\
& . \nabla^{2} \varsigma=\frac{\partial J_{1}}{\partial x}+\frac{\partial J_{3}}{\partial y}, \quad \nabla^{2} \xi=\frac{\partial J_{1}}{\partial y}-\frac{\partial J_{3}}{\partial x} .
\end{aligned}
$$

These equations will be supplemented with appropriate boundary conditions relevant to the particular application under consideration, as will be seen later on.

\section{Formulation and Solution in the Laplace and Fourier Transforms Domain}

Applying Laplace and Fourier transforms, respectively defined by the relations

$$
\begin{gathered}
\bar{f}(x, y, s)=\int_{0}^{\infty} e^{-s t} f(x, y . t) d t \\
\bar{f}^{*}(q, y, s)=\frac{1}{\sqrt{2 \pi}} \int_{-\infty}^{\infty} e^{i q z} f(x, y, s) d y .
\end{gathered}
$$

of both sides of Eqs. (24)-(26), (16), and (17) we get

$$
\begin{gathered}
{\left[\frac{d^{2}}{d y^{2}}-\left(q^{2}+\frac{i q+s}{1+\chi}\right)\right] \bar{\Phi}^{*}=-\frac{1}{1+\chi}\left[\chi \bar{\omega}^{*}+\alpha^{2} \mathrm{v}_{1} \bar{\zeta}^{*}\right],} \\
{\left[\frac{d^{2}}{d y^{2}}-\left(q^{2}+\frac{i q+2 \sigma \omega+s}{\lambda_{1}}\right)\right] \bar{\omega}^{*}-\frac{\sigma}{\lambda_{1}}\left(\frac{d^{2}}{d y^{2}}-q^{2}\right) \bar{\Phi}^{*}=0,} \\
{\left[\frac{d^{2}}{d y^{2}}-\left\{q^{2}+P_{r}\left(1+s \tau_{o}\right)(s+i q)\right\}\right] \bar{T}^{*}=0,} \\
\bar{\varsigma}^{*}=-\frac{s v_{o}^{2}}{s v_{o}^{2}+v_{1} c^{2}}\left(\bar{\Phi}^{*}+k_{o} \bar{T}^{*}\right), \\
{\left[\frac{d^{2}}{d y^{2}}-\left(q^{2}+\frac{v_{o}^{2}}{c^{2}} s^{2}+v_{1} s\right)\right] \bar{\xi}^{*}=0,}
\end{gathered}
$$

Eliminating $\bar{\varsigma}^{*}$ between Eq. (31) and (28), we obtain

$$
\begin{array}{r}
{\left[\frac{d^{2}}{d y^{2}}-\left(q^{2}+\frac{1}{1+\chi}\left\{i q+s+\frac{s v_{1} v_{o}^{2} \alpha^{2}}{s v_{o}^{2}+v_{1} c^{2}}\right\}\right)\right] \bar{\Phi}^{*}=} \\
+\frac{1}{1+\chi}\left[\chi \bar{\omega}^{*}-\frac{s v_{1} v_{o}^{2} \alpha^{2} k_{o}}{s v_{o}^{2}+v_{1} c^{2}} \bar{T}^{*}\right]=0 .
\end{array}
$$

It should be noted that this is a formal expression for the solution exponential. In the physical problem $0 \leq \mathrm{y} \leq \infty$, we should suppress the positive exponential which are unbounded at infinity. The solution of Eq. (30), and (32) can be written as

$$
\begin{aligned}
& \bar{T}^{*}=A_{1} e^{-k_{1} y}, \\
& \bar{\xi}^{*}=A_{2} e^{-k_{2} y},
\end{aligned}
$$

where $\mathrm{A}_{1}$ and $\mathrm{A}_{2}$ are some parameters depending on $\mathrm{s}$ and $\mathrm{q}$ only and

$$
\begin{gathered}
k_{1}^{2}=\left(q^{2}+P_{r}\left(1+s \tau_{o}\right)(s+i q)\right) \\
k_{2}^{2}=q^{2}+\frac{v_{o}^{2}}{c^{2}} s^{2}+v_{1} s .
\end{gathered}
$$

Eliminating $\bar{\omega}^{*}$ and $\bar{T}^{*}$ between Eqs. (29), (30) and (33) we arrive at the following fourth order differential equation satisfied by $\bar{\Phi}^{*}$

$$
\begin{aligned}
{\left[D^{4}-\left(m_{1}+m_{4}+\frac{\sigma}{\lambda_{1}} m_{2}\right) D^{2}\right.} & \left.+\left(m_{1} m_{4}+\frac{\sigma}{\lambda_{1}} q^{2}\right)\right] \bar{\Phi}^{*}= \\
& -m_{3}\left(k_{1}^{2}-m_{4}\right) A_{1} e^{-k_{1} y}
\end{aligned}
$$

where

$$
\begin{aligned}
& m_{1}=q^{2}+\frac{1}{1+\chi}\left\{i q+s+\frac{s \mathrm{v}_{1} v_{o}^{2} \alpha^{2}}{s v_{o}^{2}+v_{1} c^{2}}\right\}, m_{2}=\frac{\chi}{1+\chi}, \\
& m_{3}=\frac{s \mathrm{v}_{1} v_{o}^{2} \alpha^{2} k_{o}}{\left(s v_{o}^{2}+v_{1} c^{2}\right)(1+\chi)}, \quad m_{4}=q^{2}+\frac{i q+2 \sigma+s}{\lambda_{1}} .
\end{aligned}
$$

The solution of Eq. (36) can be written

$$
\bar{\Phi}^{*}(q, y, s)=A_{3} e^{-k_{3} y}+A_{4} e^{-k_{4} y}+\ell_{1} A_{1} e^{-k_{1} y},
$$

where $\ell_{1}=\frac{m_{3}\left(m_{4}-k_{1}^{2}\right)}{\left(k_{1}^{2}-k_{3}^{2}\right)\left(k_{1}^{2}-k_{4}^{2}\right)}$,

and $k_{3}^{2}, k_{4}^{2}$ are the roots of the characteristic equation

$$
k^{4}-\left(m_{1}+m_{4}+\frac{\sigma}{\lambda_{1}} m_{2}\right) k^{2}+\left(m_{1} m_{4}+\frac{\sigma}{\lambda_{1}} q^{2}\right)=0 .
$$

These roots are given by

$$
\begin{aligned}
& k_{3}^{2}=\left(m_{1}+m_{4}+\frac{\sigma}{\lambda_{1}} m_{2}\right)+\sqrt{\left(m_{1}+m_{4}+\frac{\sigma}{\lambda_{1}} m_{2}\right)^{2}-4\left(m_{1} m_{4}+\frac{\sigma}{\lambda_{1}} q^{2}\right)}, \\
& k_{4}^{2}=\left(m_{1}+m_{4}+\frac{\sigma}{\lambda_{1}} m_{2}\right)-\sqrt{\left(m_{1}+m_{4}+\frac{\sigma}{\lambda_{1}} m_{2}\right)^{2}-4\left(m_{1} m_{4}+\frac{\sigma}{\lambda_{1}} q^{2}\right)},
\end{aligned}
$$

Eliminating $\bar{\Phi}^{*}$ and $\bar{T}^{*}$ between Eqs. (29), (30) and (33), we find that $\bar{\omega}^{*}$ satisfy the same equation as $\bar{\Phi}^{*}$ i.e

$$
\begin{aligned}
{\left[D^{4}-\left(m_{1}+m_{4}+\frac{\sigma}{\lambda_{1}} m_{2}\right) D^{2}+(\right.} & \left.\left.m_{1} m_{4}+\frac{\sigma}{\lambda_{1}} q^{2}\right)\right] \bar{\omega}^{*}= \\
& -\frac{\sigma m_{3}}{\lambda_{1}}\left(k_{1}^{2}-q^{2}\right) A_{1} e^{-k_{1} y},
\end{aligned}
$$

The solution of Eq. (37) has the form 


$$
\bar{\omega}^{*}(q, y, s)=\ell_{3} A_{3} e^{-k_{3} y}+\ell_{4} A_{4} e^{-k_{4} y}+\ell_{2} A_{1} e^{-k_{1} y},
$$

for some parameters $\ell_{3} A_{3}, \ell_{4} A_{4}$. The compatibility between these equations and Eqs. (37) and (29), give

$$
\ell_{2}=\frac{\sigma m_{3}\left(q^{2}-k_{1}^{2}\right)}{\lambda_{1}\left(k_{1}^{2}-k_{3}^{2}\right)\left(k_{1}^{2}-k_{4}^{2}\right)}, \quad \ell_{i}=\frac{\sigma\left(k_{i}^{2}-q^{2}\right)}{\lambda_{1}\left(k_{i}^{2}-m_{4}\right)}, \quad i=3,4 .
$$

Through out the equations (34) and (37), we can obtain the other component of current density potential functions $\bar{\varsigma}^{*}$ :

$$
\bar{\varsigma}^{*}=-\ell\left[A_{3} e^{-k_{3} y}+A_{4} e^{-k_{4} y}+\left(\ell_{1}+k_{o}\right) A_{1} e^{-k_{1} y}\right],
$$

where $\ell=\frac{s v_{o}^{2}}{s v_{o}^{2}+v_{1} c^{2}}$

By Applying Laplace and Fourier transforms of both sides of Eqs (27) and by substituting into Eqs (35), (37) and (40) we can obtain the velocity and current density components as follows:

$$
\begin{gathered}
\bar{u}^{*}=-\left(k_{3} A_{3} e^{-k_{3} y}+k_{4} A_{4} e^{-k_{4} y}+k_{1} \ell_{1} A_{1} e^{-k_{3} y}\right) \\
\bar{v}^{*}=-i q\left(A_{3} e^{-k_{3} y}+A_{4} e^{-k_{4} y}+\ell_{1} A_{1} e^{-k_{1} y}\right), \\
\bar{J}_{1}^{*}=-\left[i q \ell\left\{A_{3} e^{-k_{3} y}+A_{4} e^{-k_{4} y}+\left(\ell_{1}+k_{o}\right) A_{1} e^{-k_{1} y}\right\}+k_{2} A_{2} e^{-k_{2} y}\right], \\
\bar{J}_{2}^{*}=\ell\left[k_{3} A_{3} e^{-k_{3} y}+k_{4} A_{4} e^{-k_{4} y}+\left(\ell_{1}+k_{o}\right) k_{1} A_{1} e^{-k_{1} y}\right]+i q A_{2} e^{-k_{2} y} .
\end{gathered}
$$

By using equation (23) and (35), after Applying Laplace and Fourier transforms, the fluid pressure takes the form:

$$
\bar{p}^{*}=-\alpha^{2} \mathrm{v}_{1} A_{2} e^{-k_{2} y},
$$

The induced electric field components $\mathrm{E}_{1}, \mathrm{E}_{2}$ and induced magnetic field $\mathrm{h}$ can be obtained by substituting from (50)-(54) into (20)-(22), after Applying Laplace and Fourier transforms, we get

$$
\begin{gathered}
\bar{E}_{1}^{*}=i q(1-\ell)\left[A_{3} e^{-k_{3} y}+A_{4} e^{-k_{4} y}+A_{1}\left(\ell_{1}+k_{o}\right) e^{-k_{1} y}\right]-k_{2} A_{2} e^{-k_{2} y} \\
\bar{E}_{2}^{*}=(\ell-1)\left[k_{3} A_{3} e^{-k_{3} y}+k_{4} A_{4} e^{-k_{4} y}+k_{1} A_{1}\left(\ell_{1}+k_{o}\right) e^{-k_{1} y}\right]-i q A_{2} e^{-k_{2} y} \\
\bar{h}^{*}=\left(k_{2}^{2}-q^{2}\right) A_{2} e^{-k_{2} y} .
\end{gathered}
$$

We have thus obtained the solution of the problem in the transformed domain in terms of four unknown parameters $A_{1}$, $A_{2}, A_{3}$, and $A_{4}$. These parameters are to be determined from the boundary conditions at $\mathrm{y}=0$.

\section{Applications}

We shall consider unsteady two-dimensional flow of an incompressible micropolar conducting fluid past an infinite horizontal plane surface in the presence of a induced magnetic field occupying the region $0 \leq y<\infty$ of the space. Choosing the y-axis perpendicular to the surface of the plate with the origin coinciding with the middle plate, the region $\Omega$ under consideration becomes

$\Omega=[(x, y, z):-\infty<x<\infty, 0 \leq y<\infty,-\infty<z<\infty]$

(1) Thermal boundary condition

The boundary of the half-space is affected by ramp-type heating, which depends on the coordinate $\mathrm{x}$ and the time $\mathrm{t}$ of the form

$$
T(x, 0, t)=G(t) F(x),
$$

where $F(x)$ is an arbitrary function of $x$ and $G(t)$ is a function defined as[25]

$$
G(t)= \begin{cases}0 & t \leq 0, \\ T_{1} \frac{t}{t_{o}} & 0<t \leq t_{o}, \\ T_{1} & t>t_{o},\end{cases}
$$

where $t_{0}$ indicates the length of time to rise the heat and $T_{1}$ is constant, this means that the boundary of the half-space, which is initially at rest and has a fixed temperature $T_{0}$, is suddenly raised to a temperature equal to the function $\mathrm{G}(\mathrm{t}) \mathrm{F}(\mathrm{x})$ and maintained at this temperature from then on.

Applying Laplace and Fourier transforms to both sides of Eq. (47), we obtain

$$
\bar{T}^{*}(x, q, s)=\bar{G}(s) \bar{F}^{*}(q),
$$

where

$$
\bar{G}(s)=T_{1} \frac{\left(1-e^{-s t_{o}}\right)}{t_{0} s^{2}} .
$$

(2) Mechanical boundary conditions

We assume that, the plate is moving with velocity $\mathrm{v}_{\mathrm{o}}$ and non-conducting surface. The transverse components of the electric field intensity are continuous across the surface of the half-space. This leads to:

$$
y=0, \quad \bar{u}^{*}=\bar{v}_{o}^{*}, \quad \bar{\omega}^{*}=-n \frac{d \bar{u}^{*}}{d y}, \quad \bar{E}_{1}^{*}=0 .
$$

We notice that $\mathrm{n}$ is a constant such $0 \leq n \leq 1$, where the case $\mathrm{n}=0$ is called strong concentration by Guram and Smith[22], indicates $\omega=0$ near the wall and represents concentrated particle flows in which the microelements close to the wall surface are unable to rotate (Jena and Mathur[26]). The case $n=1 / 2$ indicates the vanishing of anti-symmetrical part of the stress tensor and denotes weak concentration (Ahmadi[27]). The case $\mathrm{n}=1$, as suggested by Peddieson[28], is used for the modeling of turbulent boundary-layer flows.

With the help of equations (34), (39), (41), (44), (49) and (50) we obtained

$$
\begin{gathered}
A_{1}=\bar{G}(s) \bar{F}^{*}(q), \\
A_{2}=\frac{i q}{k_{2}}(1-\ell)\left[A_{3}+A_{4}+A_{1}\left(\ell_{1}+k_{o}\right)\right], \\
A_{3}=\frac{\mathrm{A}_{1}\left[\ell_{1} \mathrm{k}_{1}\left\{\ell_{4}+\mathrm{nk}_{4}\left(\mathrm{k}_{1}-\mathrm{k}_{4}\right)\right\}-\ell_{2} \mathrm{k}_{4}\right]+v_{o}\left(\ell_{4}-\mathrm{nk}_{4}^{2}\right)}{\ell_{3} \mathrm{k}_{4}-\mathrm{k}_{3}\left[\ell_{4}+\mathrm{nk}_{4}\left(\mathrm{k}_{3}-\mathrm{k}_{4}\right)\right]}, \\
A_{4}=\frac{\mathrm{A}_{1}\left[\ell_{1} \mathrm{k}_{1}\left\{\ell_{3}+\mathrm{nk}_{3}\left(\mathrm{k}_{1}-\mathrm{k}_{3}\right)\right\}-\ell_{2} \mathrm{k}_{3}\right]+v_{o}\left(\ell_{3}-\mathrm{nk}_{3}^{2}\right)}{\mathrm{k}_{3}\left[\ell_{4}+\mathrm{nk}_{4}\left(\mathrm{k}_{3}-\mathrm{k}_{4}\right)\right]-\ell_{3} \mathrm{k}_{4}},
\end{gathered}
$$

\section{Inversion of the Transforms}

To obtain the solution of the problem in the physical domain $(x, y, t)$, we have to invert the iterated transforms in Eqs. (41)-(46). These expressions can be formally expressed as functions of $\mathrm{x}$ and the parameter of the Fourier and Laplace transforms q and s of the form $\bar{f}^{*}(x, q, s)$. 
First, we invert the Fourier transform using the inversion formula given previously. This gives the Laplace transform expression $\bar{f}(x, q, s)$ of the function $\mathrm{f}(\mathrm{x}, \mathrm{y}, \mathrm{t})$ as

$$
\begin{aligned}
\bar{f}(x, q, t) & =\frac{1}{\sqrt{2 \pi}} \int_{-\infty}^{\infty} e^{-i q y} f(x, q, s) d q \\
& =\frac{1}{\sqrt{2 \pi}} \int_{-\infty}^{\infty}\left(\cos (q y) f_{e}+i \sin (q y) f_{o}\right) d q,
\end{aligned}
$$

where $\mathrm{f}_{\mathrm{e}}$ and $\mathrm{f}_{\mathrm{o}}$ denote the even and the odd parts of the function $\bar{f}^{*}(x, q, s)$ respectively.

We shall now outline the numerical inversion method used to find the solution in the physical domain. For fixed values of $\mathrm{x}, \mathrm{y}$, and $\mathrm{q}$ the function inside braces in the last integral can be considered as a Laplace transform $\bar{g}(s)$ of some function $g(t)$.

The inversion formula for the Laplace transform can be written as

$$
g(t)=\frac{1}{2 \pi i} \int_{c-i \infty}^{c+i \infty} e^{s t} \bar{g}(s) d s,
$$

where $\mathrm{c}$ is an arbitrary real number greater than all the parts of the singularities $\bar{g}(s)$. Taking $\mathrm{s}=\mathrm{c}+$ iy, the above integral takes the form

$$
g(t)=\frac{e^{c t}}{2 \pi} \int_{-\infty}^{\infty} e^{i y t} \bar{g}(c+i y) d y,
$$

Expanding the function $\mathrm{h}(\mathrm{t})=\exp (-\mathrm{ct}) \mathrm{g}(\mathrm{t})$ in a Fourier series in the interval[0,2L], we obtain the approximate formula.

Where

$$
g(t)=g_{\infty}(t)+E_{D},
$$

$$
g_{\infty}(t)=\frac{1}{2} c_{o}+\sum_{k=1}^{\infty} c_{k} \quad \text { for } \quad 0 \leq t \leq 2 L,
$$

and

$$
c_{k}=\frac{e^{c t}}{L} \operatorname{Re}\left[e^{i k \pi t / L} g\left(c+\frac{i k \pi t}{L}\right)\right],
$$

$E_{D}$, the discretization error, can be made arbitrary small by choosing c large enough.

Since the infinite series in Eq. (51) can be summed up to finite number $\mathrm{N}$ of terms, the approximate value of $\mathrm{g}(\mathrm{t})$ becomes

$$
g_{N}(t)=\frac{1}{2} c_{o}+\sum_{k=1}^{N} c_{k} \quad \text { for } \quad 0 \leq t \leq 2 L .
$$

Using the above formula to evaluate $\mathrm{g}(\mathrm{t})$, we introduce a truncation error $\mathrm{E}_{\mathrm{T}}$ which, must be added to the discretization error to produce the total approximation error.

Two methods are used to reduce the total error. First the "Korrecktur" method is used to reduce the discretization error. Next, the e algorithm is used to reduce the truncation error and hence to accelerate convergence.

The Korrecktur method uses the following formula to evaluate the function $g(t)$ :

$$
g(t)=g_{\infty}(t)-e^{2 c L} g_{\infty}(2 L+t)+E_{D}^{\prime},
$$

where the discretization error $\left|E_{D}^{\prime}\right|<<\left|E_{D}\right|$. Thus, the approximate value of $\mathrm{g}(\mathrm{t})$ becomes

$$
g_{N K}(t)=g_{N}(t)-e^{2 c L} g_{N^{\prime}}(2 L+t) .
$$

$\mathrm{N}^{\prime}$ is an integer such that $\mathrm{N}^{\prime}<\mathrm{N}$.

We shall now describe the e-algorithm that is used to accelerate the convergence of the series in Eq. (53).

Let $\mathrm{N}=2 \mathrm{q}+1$ where $\mathrm{q}$ is a natural number, and let

$$
s_{m}=\sum_{k=1}^{m} c_{k}
$$

be the sequence of partial sums of Eq. (53). We define the e-sequence by

$$
\varepsilon_{o, m}=0, \quad \varepsilon_{1, m}=0
$$

and

$$
\varepsilon_{p+1, m}=\frac{\varepsilon_{p-1, m+1}+1}{\varepsilon_{p, m+1}-\varepsilon_{p, m}}, \quad p=1,2,3, \ldots
$$

it can be shown that the sequence $\varepsilon_{1,1}, \varepsilon_{3,1}, \varepsilon_{5,1}, \ldots, \varepsilon_{\mathrm{N}, 1}$, converges to $f(x, y, t)+E D-c_{o} / 2$ faster than the sequence of partial sums $\mathrm{s}_{\mathrm{m}}(\mathrm{m}=1,2,3, \ldots)$.

The actual procedure used to invert the Laplace transform consists of using Eq. (67) together with the $\varepsilon$-algorithm. The values of $\mathrm{c}$ and $\mathrm{L}$ are chosen according to the criteria outlined Honig and Hirdes[29].

\section{Results and Discussion}

The purpose of this section is to see the influence of some interesting parameters on the horizontal, normal velocity, microrotation and transverse electric fields. In particular, attention has been focused to modified Ohm's law $\left(\mathrm{k}_{\mathrm{o}}\right.$ parameter) and Alfven velocity parameter $\alpha$ in two cases the first for strong concentration $(\mathrm{n}=0)$ and the other for weak concentration $(n=1 / 2)$, many previous studies of conventional and conjugate problems did not consider this parameter, on the horizontal and normal velocity. The effect of Prandtl number $P_{r}$, ramp-type heating parameter $t_{o}$ and modified Fourier law's (relaxation time $\tau_{\mathrm{o}}$ ) are also investigated on the temperature. The distribution of non- dimensional temperature $\mathrm{T}$, non-dimensional horizontal velocity $\mathrm{u}$, non-dimensional normal velocity $\mathrm{v}$, non-dimensional micro-rotation $\omega$, and non-dimensional transverse electric field $\mathrm{E}_{1}$ with non-dimensional distance y have been shown in Figs. $1-12$. Numerical analysis has been carried out by taking $\mathrm{x}$ range from 0 to 1 for strong concentration and from 0 to 3 for weak concentration. The computations are carried out for the non-dimensional time $\mathrm{t}=1.5$ and non-dimensional $\mathrm{x}=2$.

The field quantities, velocity, microrotation and electric fields do not depend only on the state and space variables $t$, $\mathrm{x}$ and $\mathrm{y}$, but also depend on $\mathrm{k}_{\mathrm{o}}$ and Alfven velocity parameters. It has been observed that, these parameters play a vital role on velocity, microrotation and electric fields. The numerical values for the field temperature are computed for a wide range of values of finite pulse rise-time $t_{0}$ in the two 
situations $\mathrm{t}>\mathrm{t}_{\mathrm{o}}$ and $\mathrm{t}<\mathrm{t}_{\mathrm{o}}$ and relaxation time $\tau_{\mathrm{o}}=0.2$.

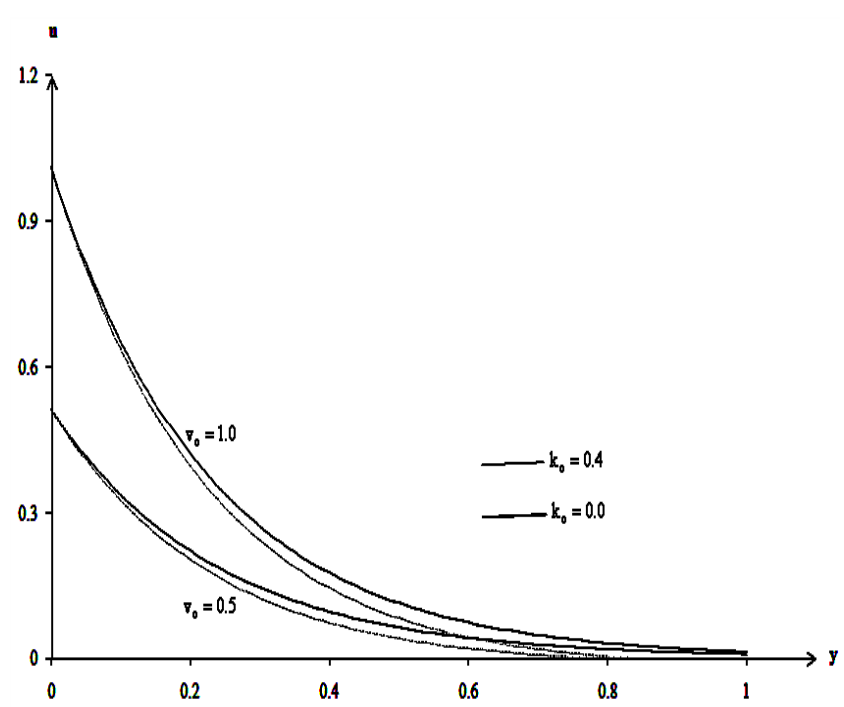

Figure 1. Horizontal velocity profiles vs. $y$ for different values of $\mathrm{k}_{0}$ and $\mathrm{v}_{0}$ for strong concentration $(\mathrm{n}=0)$

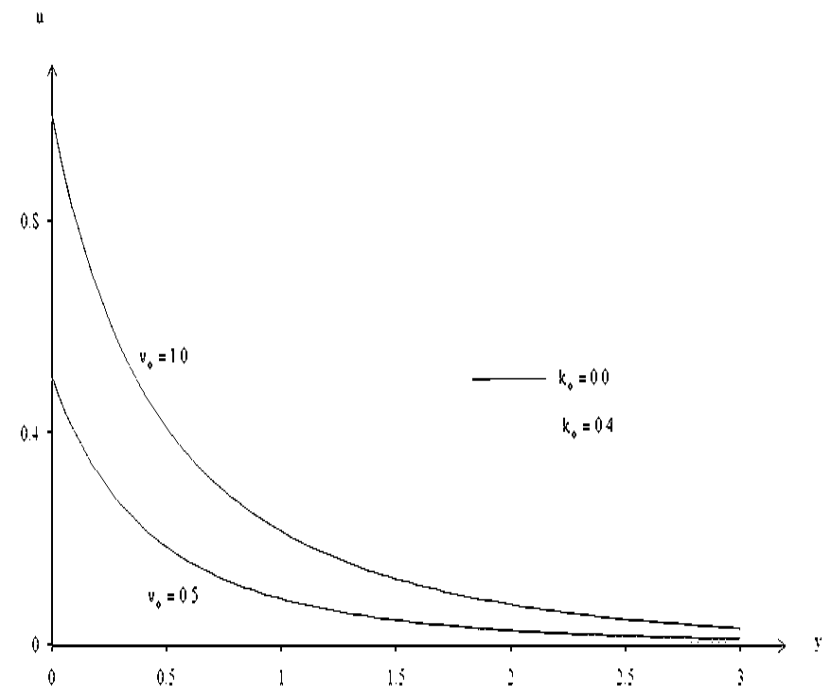

Figure 2. Horizontal velocity profiles vs. $\mathrm{y}$ for different values of $\mathrm{k}_{0}$ and $\mathrm{v}_{0}$ for weak concentration $(\mathrm{n}=1 / 2)$

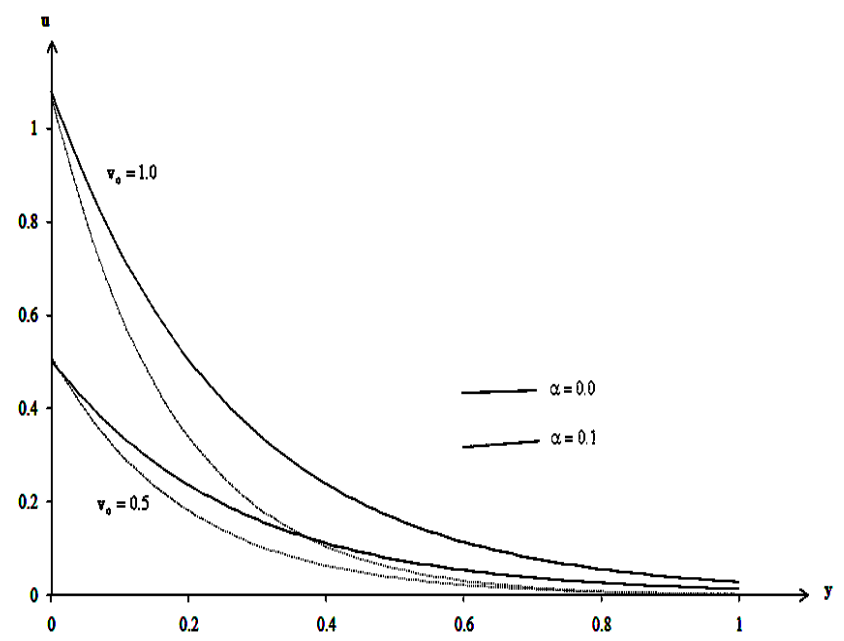

Figure 3. Horizontal velocity profiles vs. y for different values of a and $\mathrm{v}_{0}$ for strong concentration $(\mathrm{n}=0)$

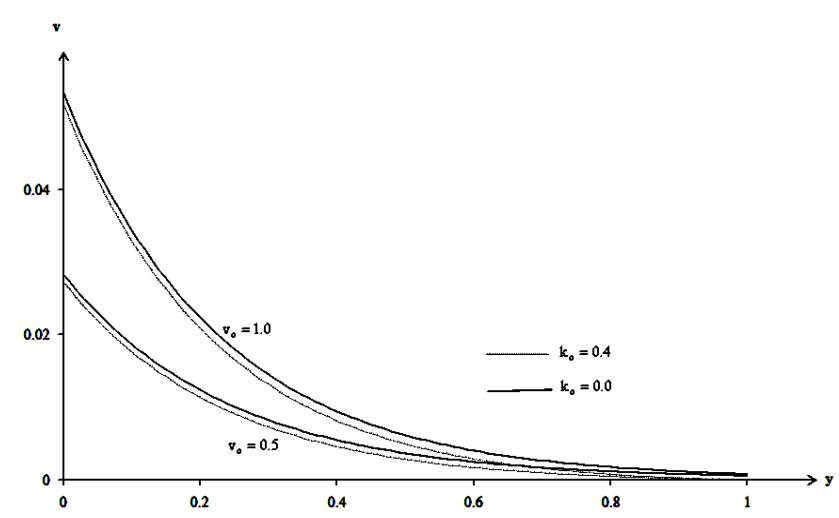

Figure 4. Normal velocity profiles vs. $y$ for different values of $\mathrm{k}_{0}$ and $\mathrm{v}_{0}$ for strong concentration $(\mathrm{n}=0)$

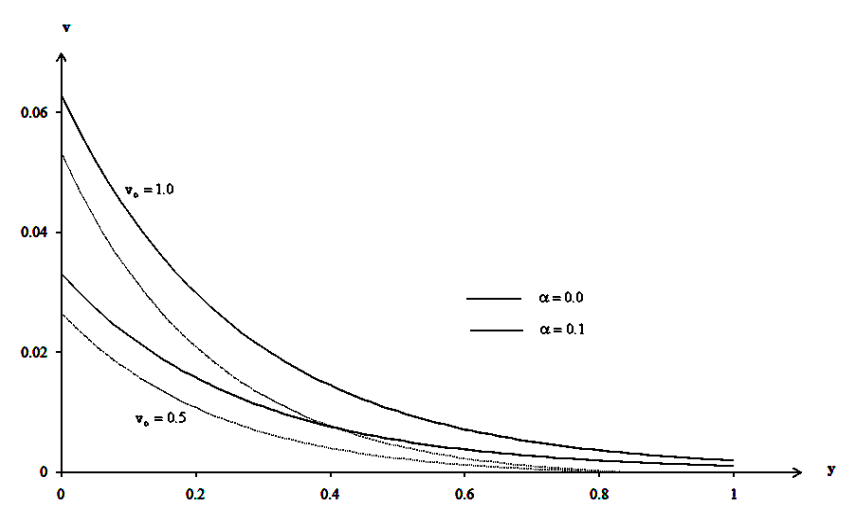

Figure 5. Normal velocity profiles vs. $y$ for different values of $a$ and $v_{0}$ for strong concentration $(\mathrm{n}=0)$

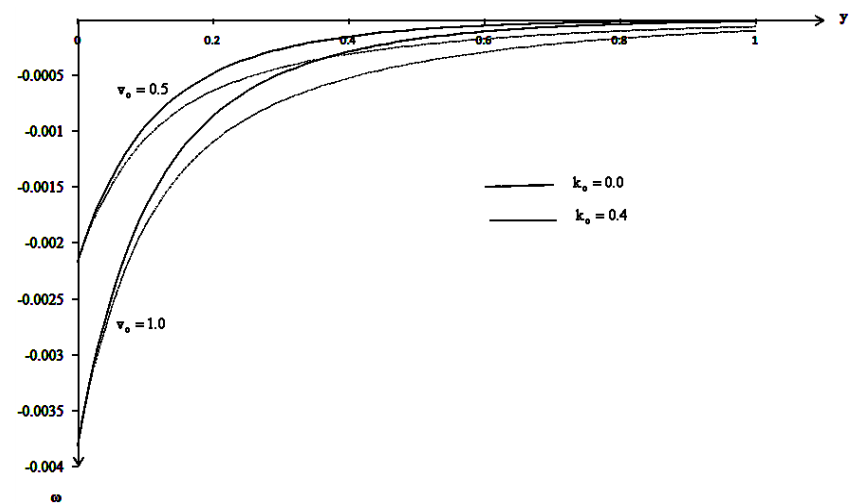

Figure 6. microrotation profiles vs. $\mathrm{y}$ for different values of $\mathrm{k}_{0}$ and $\mathrm{v}_{0}$ for strong concentration $(\mathrm{n}=0)$

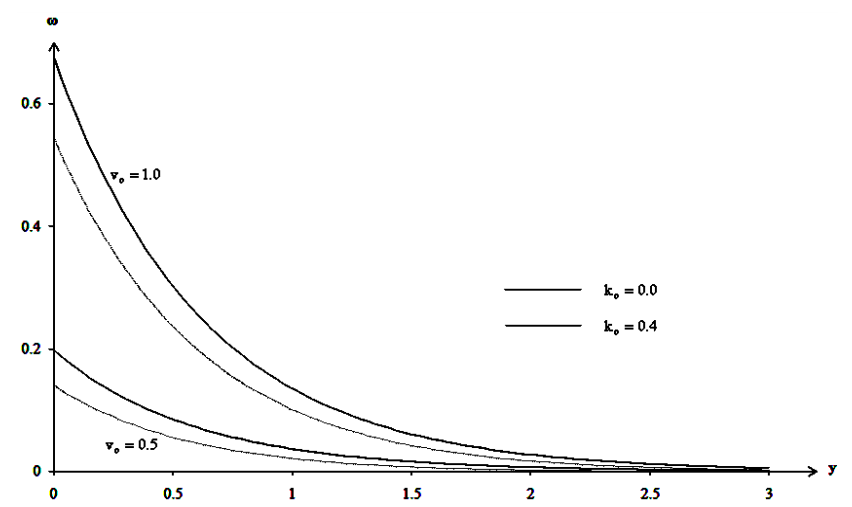

Figure 7. microrotation profiles vs. $\mathrm{y}$ for different values of $\mathrm{k}_{0}$ and $\mathrm{v}_{0}$ for weak concentration $(n=1 / 2)$ 


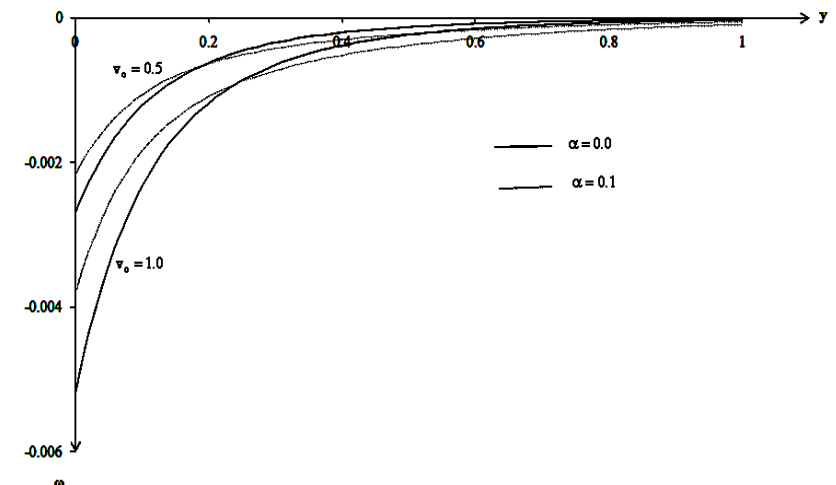

Figure 8. microrotation profiles vs. $y$ for different values of $a$ and $v_{0}$ for strong concentration $(\mathrm{n}=0)$

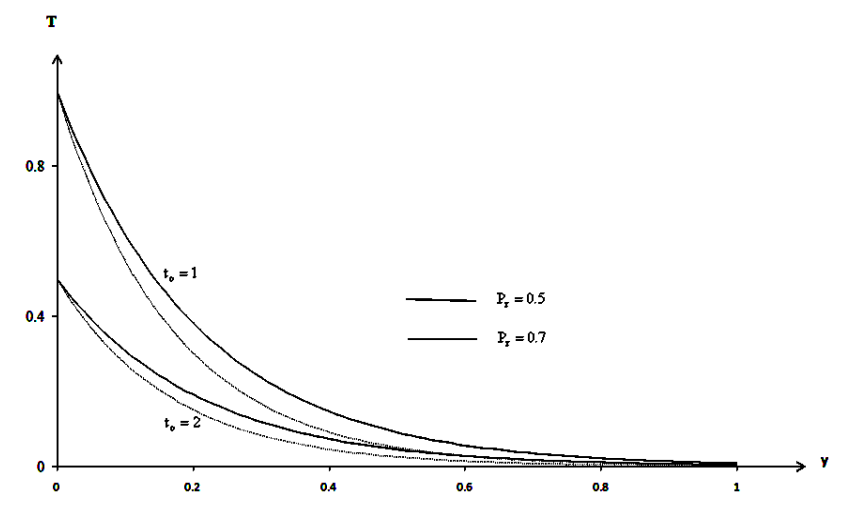

Figure 9. Temperature profiles vs. $y$ for different values of $P_{r}$ and $t_{o}$ for strong concentration $(\mathrm{n}=0)$

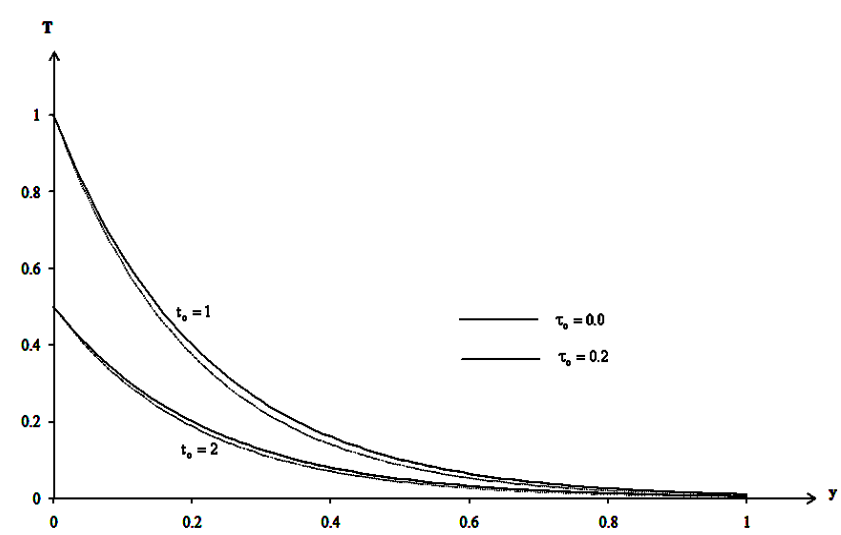

Figure 10. Temperature profiles vs. $y$ for different values of $\tau_{0}$ and $t_{0}$ for strong concentration $(\mathrm{n}=0)$

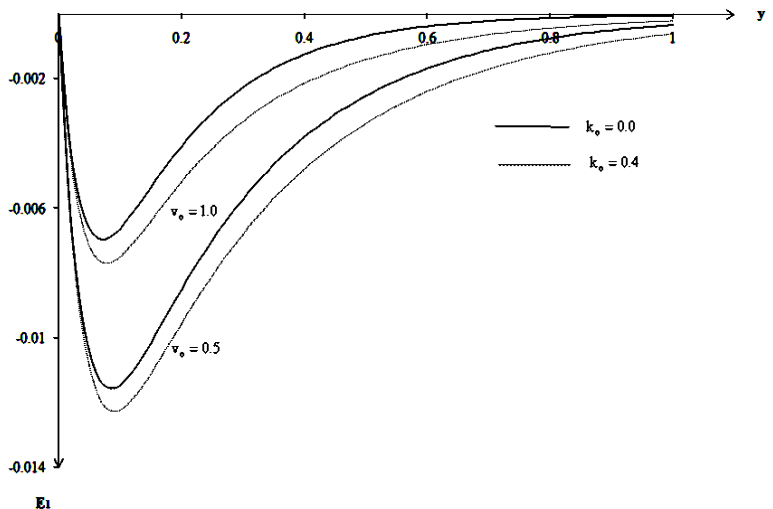

Figure 11. Transverse electric field profiles vs. $y$ for different values of $k_{o}$ and $\mathrm{v}_{\mathrm{o}}$ for strong concentration $(\mathrm{n}=0)$

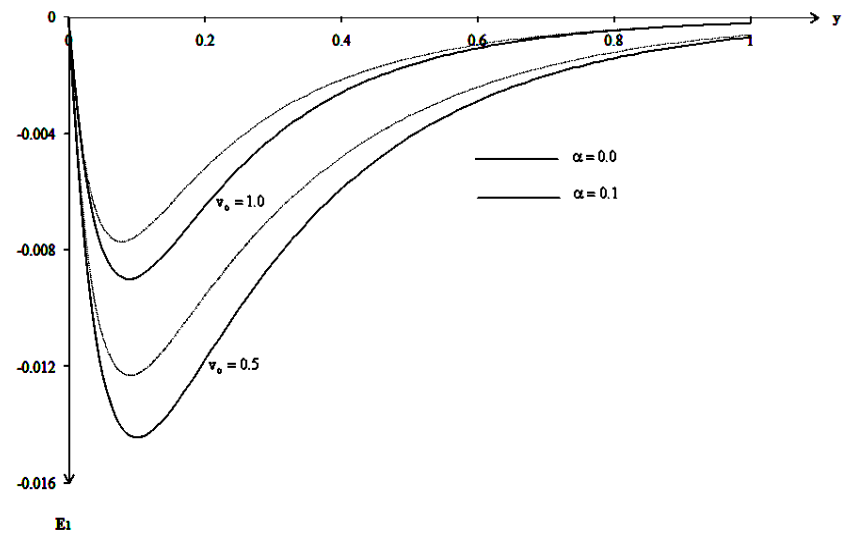

Figure 12. Transverse electric field profiles vs. $y$ for different values of a and $\mathrm{v}_{\mathrm{o}}$ for strong concentration $(\mathrm{n}=0)$

The solid line represents horizontal velocity for electromagnetic micropolar with modified Ohm's law effect $\left(\mathrm{k}_{\mathrm{o}}=0.4\right)$ when $\mathrm{v}_{\mathrm{o}}=1.0$ or $\mathrm{v}_{\mathrm{o}}=0.5$, while small dotes line represents electromagnetic micropolar without modified Ohm's law effect $\left(\mathrm{k}_{\mathrm{o}}=0.0\right)$ in figures 1 and 2 , we consider the strong concentration $(\mathrm{n}=0)$ case in figure 1 and weak concentration $(n=1 / 2)$ case in figure 2 . It is seen that these profiles develop rapidly as increases from boundary condition for $\mathrm{v}_{\mathrm{o}}$ and they attain the steady-state flow at $\mathrm{y} \rightarrow 1$ for $\mathrm{n}=0$ or $\mathrm{y} \rightarrow 3$ for $\mathrm{n}=1 / 2$ and, also note that the horizontal velocity becomes finite speed in strong concentration case as compared to weak concentration case. Finally it is obvious that the values of horizontal are decreased as $\mathrm{k}_{\mathrm{o}}$ parameter increases, and then tends to zero.

Fig. 3 is prepared to show the effect of the variations of Alfven velocity $\alpha$ and $v_{0}$. We can see that an increase in $\alpha$ (or decrease in $v_{0}$ ) decreases the horizontal velocity profiles.

For different values of the gradient temperature parameter $\mathrm{k}_{\mathrm{o}}$ and Alfven velocity parameter $\alpha$, the normal velocity profiles are plotted in figures 4 and 5 . It is obvious that the effect of increasing values of $k_{0}$-parameter and $\alpha$-parameter results in a decreasing velocity distribution across flow of the micropolar fluid. The results also show that the magnitude of normal velocity on the plate is decreased as $\mathrm{v}_{0}$-parameter increases.

Figs. 6, 7 elucidate the variations of $\mathrm{k}_{0}$ coefficient and the plate velocity $\mathrm{v}_{\mathrm{o}}$ against the dimensionless $\mathrm{y}$ for different cases of the microrotation profiles (strong and weak concentration). The range of the dimensionless $\mathrm{k}_{\mathrm{o}}$ parameter is considered from 0 to 0.4 (corresponding to the classical and modified Ohm's law. This figures show that increasing the value of the modified Ohm's law reaction parameter $k_{o}$ decreases the value of the microrotation distribution due to the lost heat generating from the movement of electric current, this heat may be the main reason to make that the deformation of the medium tends to be normal, but the rotation of the particles becomes slower than that in the old model. Also, it is predicted that the microrotation rate enhances while the coefficient of plate velocity increases in strong concentration but decrease in weak concentration. The sample of microrotation profiles (strong concentration) for various values of Alfven velocity parameter $\alpha$ when $\mathrm{v}_{0}=0.5$ and 1.0 is dis- 
played in Fig. 8. It is observed that the absolute value of the dimensionless microrotation or angular velocity $|\omega(\mathrm{y})|$ continuously decreases with y and becomes zero far away from the surface, which satisfies the boundary conditions (50). As expected, the microrotation effects are more dominant near the wall. Also, $|\omega(\mathrm{y})|$ increases as $\alpha$ and $\mathrm{v}_{\mathrm{o}}$ increase in the vicinity of the surface but the reverse happens as one moves away from it.

Figs.9 and 10 illustrate the temperature profiles for different values of Prandtl number Pr and modified Fourier's law parameter $\tau_{0}$ for two values of $t_{0}$, respectively. The numerical results show that the effect of increasing values of Prandtl number results in a decreasing temperature, while the inclusion of the relaxation time modifies the governing thermal equation, changing it from the parabolic to a hyperbolic type, and thereby eliminating the unrealistic result that thermal disturbance is realized instantaneously everywhere within a fluid and . From Fig.9, it is observed that an increase in the Prandtl number results a decrease of the boundary thermal thickness of the fluid and in general lower average temperature within the fluid. The reason is that smaller values of $\mathrm{P}_{\mathrm{r}}$ are equivalent to increasing the thermal conductivities, and therefore heat is able to diffuse away from the heated surface more rapidly than for higher values of $\mathrm{P}_{\mathrm{r}}$. Also it is observed that the significant difference in the value of temperature is noticed for different value of the ramping time parameter $t_{0}$ in the context of the old and new models. We noticed that, the temperature increases when $\mathrm{t}_{\mathrm{o}}<$ $\mathrm{t}\left(1<\mathrm{t} / \mathrm{t}_{0}\right)$ and decreases when $\mathrm{t}_{\mathrm{o}}>\mathrm{t}\left(\mathrm{t} / \mathrm{t}_{\mathrm{o}}<1\right)$ which we can see that from the boundary condition (48).

Finally, the profiles of the transverse electric field $E_{1}$ as a function of all the parameters of the modified Ohm's law $k_{0}$ and Alfven velocity $\alpha$ treated here, have been in shown figures $(11,12)$. From these figures we observe that the effect of $\mathrm{k}_{\mathrm{o}}$ is to decrease the transverse electric field, while the effect of Alfven velocity $\alpha$ is to increase it in strong concentration case.

\section{Conclusions}

The trend of variations of the temperature distribution $\mathrm{T}$, horizontal velocity $\mathrm{u}$, normal velocity $\mathrm{v}$, horizontal conduction current density component $\mathrm{J} 1$ normal conduction current density component $\mathrm{J} 2$, and microrotation vector $\omega$ are quite different on the application of new model and old model. The medium which are taken is affected by parameters ko, $\tau$ o and magnetic field, more on the application of modified Ohm's and Fourier's laws in comparison to the application of classical model. The increasing in the values of temperature may be explained as the lost heat generating from the movement of electric current, this heat may be the main reason to make that the deformation of the micropolar fluid tends to be normal while the components of electric and magnetic fields record values greater than the values recorded in the old model.

\section{Nomenclature}

$\nabla$ del operator

H magnetic intensity vector

J conduction current density vector

D electric flux vector

E electric intensity vector

B magnetic flux vector

$\rho_{\mathrm{e}} \quad$ charge density

$\mu_{0} \quad$ magnetic permeability

$\varepsilon_{\mathrm{o}} \quad$ electric permittivity

$\sigma_{0} \quad$ electric conductivity

V velocity vector

$\mathrm{T}$ temperature of the fluid

$T_{0}$ mean temperature of the surface

$\rho$ density of the fluid

$\mathrm{C}_{\mathrm{E}}$ specific heat at constant pressure

$\lambda$ thermal conductivity

q heat flux vector

$\tau_{0} \quad$ relaxation time

$\mu \quad$ shear kinematic viscosity

b body force per unit mass

$\omega$ microrotation vector

j gyration parameters

$\mu_{\mathrm{r}} \quad$ coupling viscosity coefficient or vortex viscosity

I body couple per unit mass

$\alpha^{2}=\frac{\mu_{o} H_{o}^{2}}{\rho}$, Alfven velocity

$v \quad$ kinematic viscosity

$v_{\mathrm{r}} \quad$ kinematic coupling viscosity

$T_{\infty}$ temperature far away from the surface

$\mathrm{p}$ fluid pressure

U free stream velocity

$\mathrm{P}_{\mathrm{r}} \quad$ Prandtl number

c $\quad$ speed of light $=1 / \sqrt{\varepsilon_{0} \mu_{o}}$

\section{REFERENCES}

[1] Papautsky, J. Brazzle, T. Ameel, A. B. Frazier, Laminar fluid behavior in microchannels using micropolar fluid theory. Sensors Actuators A Physical (1999) 73(1-2):101-108.

[2] J. J. Shu , Microscale heat transfer in a free jet against a plane surface. Superlattices Microstruct (2004) 35: 645-656.

[3] C. Eringen, Microcontinuum field theories II: fluent Media. Springer-Verlag, New York, Inc (2001).

[4] S. Srinivas, M. Kothandapani: The influence of heat and mass transfer on MHD peristaltic flow through a porous space with compliant walls, App. Math. Comp. 213 (2009) 197-208.

[5] Chien-Hsin: Magneto-hydrodynamic mixed convection of a power-law fluid past a stretching surface in the presence of thermal radiation and internal heat generation/absorption, Int. J. Non-Linear Mech. 44 (2009) 596-603.

[6] S. Srinivas, M. Kothandapani: The influence of heat and mass transfer on MHD peristaltic flow through a porous 
space with compliant walls, Appl. Math. Comp.213 (2009) 197-208

[7] R. C. Chaudhary, Abhay Kumar Jha: Effects of chemical reactions on MHD micropolar fluid flow past a vertical plate in slip-flow regime, Appl. Math. Mech. -Engl. Ed., 2008, 29(9):1179-1194.

[8] Youn J. Kim: Heat and Mass Transfer in MHD Micropolar Flow Over a Vertical Moving Porous Plate in a Porous Medium, Transport in Porous Media 56: 17-37, 2004.

[9] Ishak , R. Nazar , I. Pop: MHD boundary-layer flow of a micropolar fluid past a wedge with variable wall temperature, Acta Mech 196, 75-86 (2008)

[10] Ishak, R. Nazar, I. Pop: Mixed convection stagnation point flow of a micropolar fluid towards a stretching sheet, Meccanica (2008) 43: 411-418.

[11] F. Ali, M. Norzieha, S. Sharidan, I. Khan, T. Hayat: New exact solutions of Stokes' second problem for an MHD second grade fluid in a porous space, Int. J. of Non-Linear Mech. In Press.

[12] Mostafa A.A. Mahmoud: Thermal radiation effects on MHD flow of a micropolar fluid over a stretching surface with variable thermal conductivity, Physica A 375 (2007) 401-410.

[13] M. Ezzat, A.A. El-Bary, S. Ezzat: Combined heat and mass transfer for unsteady MHD flow of perfect conducting micropolar fluid with thermal relaxation, Energy Conversion and Management, 52(2011) $934-945$.

[14] Mustafa Turkyilmazoglu: Effects of uniform radial electric field on the MHD heat and fluid flow due to a rotating disk Original Research Article, Int. J. Eng. Sci., 51 (2012) 233 240.

[15] K. Das: Effect of chemical reaction and thermal radiation on heat and mass transfer flow of MHD micropolar fluid in a rotating frame of reference, International Journal of Heat and Mass Transfer, Int. J. Heat and Mass Trans., 54 (2011) 3505 3513.

[16] C. Eringen, Theory of thermo-stretch fuids and bubbly liquids. Int J Eng Sci (1990) 28: 133-143

[17] M. Zakaria, Problem in electromagnetic free convection flow of a micropolar fluid with relaxation time through a porous medium. Appl. Math. Comp. 151 (2004) 601-613.

[18] M. Ezzat and M. Zakaria, Heat transfer with thermal relaxation to a perfectly conducting polar fluid, Heat Mass Transfer $41(2005) 189$.

[19] M. Zakaria, Magnetohydrodynamic unsteady free convection flow of a couple stress fluid with one relaxation time through a porous medium, J. Appl. Math. comp 146 (2003) 469.

[20] M. Ezzat, M. Zakaria, and M. Moursy, Magneto-hydrodynamic boundary layer flow past a stretching plate and heat transfer, J. Appl. Math. 1 (2004) 9.

[21] C. Eringen, Electrodynamics of microstretch and micropolar fluids. ARI (1998) 50: 169-179

[22] H. Nayfeh, S. Nemat-Nasser, Electromagneto- thermoelastic plane waves in solids with thermal relaxation, J Applied Mech, March (1972)108-113.

[23] T. Buchukuri, R. Chichinadze, Two-dimensional problems of satationary flow of a noncompressible viscous fluid in the case of Oseens's linearlization, Georgian Mathematical Journal 1(1994), No. 3, 251-266

[24] W.E. Olmstead, fundamental Oseen solution for the 2-dimensional flow of a micropolar fluid. Int. J. Engng. (1983) 21: 423-430.

[25] G.S. Guram, A.C. Smith, Stagnation flow of micropolar fluids with strong and weak interactions, Comput. Math. Appl. (1980) 6: 213-233.

[26] S.K. Jena, M.N. Mathur, Similarity solutions for laminar free convection flow of a thermo-micropolar fluid past a non-isothermal vertical flat plate, Int. J. Eng. Sci. (1981) 19: 1431-1439.

[27] G. Ahmadi, Self-similar solution of incompressible micropolar boundary layer flow over a semi-infinite plate, Int. J. Eng. Sci. (1976) 14: 639-646.

[28] J. Peddieson, An application of the micropolar fluid model to the calculation of turbulent shear flow, Int. J. Eng. Sci. (1972) 1010: 23-32.

[29] G. Honig, U. Hirdes, A method for the numerical inversion of Laplace transform. J. Comp. Appl. Math. 10 (1984) 113-132. 